\title{
Epilepsy surgery in childhood: no longer the treatment of last resort
}

\author{
George M. Ibrahim MD, James T. Rutka MD PhD, O. Carter Snead III MD
}

See related review by Jette and colleagues on page 997 and at www.cmaj.ca/lookup/doi/10.1503/cmaj.121291

$\mathrm{E}$ pilepsy occurs in $1 \%-2 \%$ of children and has been identified by the World Health Organization as the most common serious neurologic condition affecting children. ${ }^{1} \mathrm{Up}$ to one-third of patients with epilepsy do not respond to medical therapy, which is defined as failure of two appropriately selected antiepileptic drugs to control seizures at maximally tolerated serum levels for two years. ${ }^{1}$ Individuals who do not respond to two appropriately selected antiepileptic drugs have less than a $5 \%$ chance of responding to additional medical treatments. ${ }^{2}$ In an accompanying review, Jette and colleagues ${ }^{3}$ examine evidence showing improved rates of freedom from seizures following surgery in appropriately selected pediatric and adult populations. Surgical treatments for medically refractory epilepsy in children are, however, underused in Canada, with prolonged delays in referral for presurgical evaluation. ${ }^{4}$

We explore three questions: Why is intractable epilepsy in childhood a problem? What evidence supports referral for surgical evaluation? And what are the challenges and possible solutions to increasing access to surgical care for affected children in Canada?

Intractable epilepsy is associated with increased mortality, poor quality of life, cognitive decline and high personal and societal costs. ${ }^{3}$ As outlined by Jette and colleagues, ${ }^{3}$ there is an added concern about the detrimental effects of recurrent seizures among children, as well as the prolonged use of antiepileptic drugs on the developing brain and, hence, the neurodevelopmental trajectory. Animal models have shown consistently that although the immature brain may be more resistant to cellular loss following prolonged seizure activity than the adult brain, recurrent seizures during brain development result in major changes in synaptic network organization and irreversible alterations in neuronal connectivity. ${ }^{5}$ These data indicate that ongoing seizures in children may be partially responsible for the observation that nearly half of all children with intractable epilepsy have high rates of comorbid learning difficulties, developmental delay, psychiatric and behavioural challenges and psychosocial problems. ${ }^{6}$ Indeed, worsening cognition is associated with increasing severity, frequency and duration of seizures, and younger age at seizure onset.?

Given the considerable medical and psychosocial burden associated with intractable epilepsy in childhood, it would appear that potentially curative surgical treatments that can alter the natural history of the disease should be considered sooner rather than later. Jette and colleagues review available randomized controlled trials that have validated the benefits of surgical intervention over standard medical therapy for adults with medically intractable temporal lobe epilepsy. ${ }^{3}$ Although no trial evidence exists for children, in appropriately selected cohorts with seizure foci arising both within and outside the temporal lobe, the rate of surgical cure may vary from two-thirds to three-quarters. ${ }^{6}$ With advanced diagnostic tools, surgical cure may also be achieved for children without an obvious structural lesion (i.e., no lesion visible on magnetic resonance imaging or nonlesional epilepsy) and those with multilobar or widespread epileptic networks. The review by Jette and colleagues ${ }^{3}$ emphasizes that patients with nonlocalized epilepsy may also benefit from "palliative" surgical procedures such as neuromodulation, including vagus nerve and deep brain stimulation to decrease seizure frequency or severity without curative intent. A reduction in seizure frequency

\section{- KEY POINTS}

- Childhood epilepsy is associated with a considerable medical and psychosocial burden and adversely affects the developing brain.

- The best available evidence indicates that epilepsy surgery during childhood is a safe and effective treatment, yet few children who may be eligible for epilepsy surgery in Canada are currently being evaluated for surgical candidacy.

- Increased surgical capacity and awareness of epilepsy surgery in childhood among clinicians and patients are necessary to improve access to presurgical evaluation. 
has been associated with improved quality of life ${ }^{8}$ and improved provision of care to children by caregivers. ${ }^{9}$ Surgical treatment may positively affect developmental and neurocognitive outcomes, particularly in cases of hemispheric epilepsy syndromes. ${ }^{6}$

The crux of the problem is, therefore, the dichotomy between the known natural history of intractable childhood epilepsy with all of its disabling comorbidities and the increasing recognition of the benefits of surgical intervention on the one hand and a lack of momentum to refer children for presurgical evaluation on the other. Currently, few children in Ontario are referred for presurgical evaluation, with a mean delay between first seizure and referral for evaluation of surgical candidacy of 4.6 years and up to 16 years. ${ }^{4}$ Indeed, adults, who in some circumstances face referral delays of over 20 years for surgically remediable temporal lobe epilepsy, may have also benefited from surgical evaluation as children. With surgical treatment based on appropriate and rigorous presurgical diagnostic evaluation, these individuals may have been spared years of medical complications, psychological morbidity, poor employment prospects and social isolation.

Various investigators have sought reasons for the discordance between clinical practice and the best available medical evidence. Epilepsy surgery is underutilized in children for several reasons, which include a lack of awareness among family practitioners, pediatricians and neurologists of the potential benefits of surgical intervention and misconceptions about the risk-to-benefit profiles. ${ }^{10}$ Other barriers to access include a perceived lack of communication between large referral centres and the community at large, and the hindrance created by the severe underlying disability for the patients, which may prevent them (or their families) from advocating for surgical treatment. ${ }^{11}$ To circumvent these challenges, greater emphasis and increased awareness and education among family practitioners, pediatricians, internists and neurologists about the potential benefit of epilepsy surgery over continued medical management in children is critical. ${ }^{10}$ Stronger collaborations are required between adult and pediatric clinicians as well as between medical and surgical teams. Larger referral centres performing epilepsy surgery must also en- gage with the local community to optimize bidirectional communication. In this regard, new tools are emerging to guide appropriate referrals for epilepsy surgery. ${ }^{12}$

The best available evidence suggests that the failure to refer children with medically intractable epilepsy for surgical consideration may subject them to unnecessary disability and suffering. By placing greater emphasis on the provision of the infrastructure to facilitate surgical evaluation and increasing awareness and education among first-line health care workers, medical jurisdictions within Canada may increase access to potentially curative treatments and dramatically alter the trajectory of a child's life. In the current medical climate, it is no longer acceptable to view epilepsy surgery during childhood as a treatment of last resort.

\section{References}

1. Snead OC. Surgical treatment of medically refractory epilepsy in childhood. Brain Dev 2001;23:199-207.

2. Kwan P, Brodie MJ. Early identification of refractory epilepsy. N Engl J Med 2000;342:314-9.

3. Jette N, Reid AY, Wiebe S. Surgical management of epilepsy. CMAJ 2014;186:997-1004

4. Lim ME, Bowen JM, Snead OC III, et al. Access to surgery for paediatric patients with medically refractory epilepsy: a systems analysis. Epilepsy Res 2013;107:286-96.

5. Holmes GL. Effects of seizures on brain development: lessons from the laboratory. Pediatr Neurol 2005;33:1-11.

6. Spencer S, Huh L. Outcomes of epilepsy surgery in adults and children. Lancet Neurol 2008;7:525-37.

7. Vendrame M, Alexopoulos AV, Boyer K, et al. Longer duration of epilepsy and earlier age at epilepsy onset correlate with impaired cognitive development in infancy. Epilepsy Behav 2009;16:431-5.

8. Elliott I, Kadis DS, Lach L, et al. Quality of life in young adults who underwent resective surgery for epilepsy in childhood. Epilepsia 2012;53:1577-86.

9. Ibrahim GM, Fallah A, Snead OC III, et al. Ethical issues in surgical decision making concerning children with medically intractable epilepsy. Epilepsy Behav 2011;22:154-7.

10. Erba G, Messina P, Pupillo E, et al.; OPTEFF Pediatric Group. Acceptance of epilepsy surgery in the pediatric age - what the parents think and what the doctors can do. Epilepsy Behav 2013; 29:112-20.

11. Swarztrauber K. Barriers to the management of patients with surgically remediable intractable epilepsy. CNS Spectr 2004;9: 146-52.

12. Bianchin MM, Abujamra AL, Jette N, et al. Development of an online tool to determine appropriateness for an epilepsy surgery evaluation. Neurology 2013;80:2169.

Affiliations: Division of Neurosurgery (Ibrahim, Rutka) and Division of Neurology (Snead), Hospital for Sick Children; Department of Surgery (Ibrahim, Rutka) and Institute of Medical Science (Ibrahim, Snead), University of Toronto, Toronto, Ont.

Contributors: All of the authors conceived the manuscript. George Ibrahim drafted the manuscript, which was revised by James Rutka and Carter Snead. All of the authors approved the final version submitted for publication. 